\section{Obesidade e excesso de peso em adultos indígenas Xukuru do Ororubá, Pernambuco, Brasil: magnitude, fatores socioeconômicos e demográficos associados}

\author{
Obesity and overweight in adult Xukuru of \\ Ororubá Indians, Pernambuco State, Brazil: \\ magnitude and associated socioeconomic \\ and demographic factors
}

Thatiana Regina Fávaro 1 Ricardo Ventura Santos 1 Geraldo Marcelo da Cunha 1 Iuri da Costa Leite 1 Carlos E. A. Coimbra Jr. ${ }^{1}$

\footnotetext{
1 Escola Nacional de Saúde Pública Sergio Arouca Fundação Oswaldo Cruz, Rio de Janeiro, Brasil.

Correspondência T. R. Fávaro

Departamento de Endemias Samuel Pessoa, Escola Nacional de Saúde Pública Sergio Arouca, Fundação Oswaldo Cruz.

Rua Leopoldo Bulhões 1408, Rio de Janeiro, $R J$ 21041-210, Brasil. thatifavaro@gmail.com
}

\begin{abstract}
This cross-sectional study focused on the epidemiology of overweight and obesity and the association with demographic and socioeconomic variables in a sample of 794 Xukuru of Ororubá adults 19-59 years of age, from an indigenous reserve in Pesqueira County, Pernambuco State, Brazil. Descriptive analyses and multivariate logistic regression were carried out, using cutoff points of BMI $>24.99 \mathrm{~kg} / \mathrm{m}^{2}$ for overweight and $>29.99 \mathrm{~kg} / \mathrm{m}^{2}$ for obesity. Prevalence rates of overweight and obesity were higher in women (52.2\% and $21 \%$, respectively) than in men (44.1\% and $7.5 \%$, respectively). Female sex and age (> 30 years) were associated with both outcomes in the multivariate regression. For obesity, the following variable showed statistically significant associations: socioeconomic status and the interaction between male gender and per capita income. As in other indigenous populations in Brazil, the study's findings suggest that the Xukuru are experiencing a rapid nutritional transition.
\end{abstract}

Obesity; Body Mass Index; Nutrition Surveys; South American Indians

\section{Resumo}

Este estudo transversal visa a descrever a distribuição de excesso de peso e obesidade e sua associação com variáveis demográficas e socioeconômicas entre 794 adultos indígenas, de 19 a 59 anos, da etnia Xukuru do Ororubá, povo indígena cujas terras estão localizadas no $\mathrm{Mu}$ nicípio de Pesqueira, agreste de Pernambuco, Brasil. A análise da associação entre as variáveis de desfecho, excesso de peso (IMC $>24,99 \mathrm{~kg} / \mathrm{m}^{2}$ ) e obesidade (>29,99kg/m²) e as variáveis explicativas foi realizada utilizando-se regressão logística multinível. Entre as mulheres, 52,2\% estavam com excesso de peso e $21 \%$ obesas. $\mathrm{Pa}$ ra os homens, as prevalências foram de $44,1 \% e$ $7,5 \%$, respectivamente. As variáveis sexo feminino e idade (> 30 anos) estiveram associadas à ocorrência de ambos os agravos. Status socioeconômico e interação sexo masculino e renda per capita elevada apresentaram associação com obesidade. Assim como observado em outras populações indígenas, os achados sugerem que os Xukuru estão atravessando um acelerado processo de transição nutricional.

Obesidade; Índice de Massa Corporal; Inquéritos Nutricionais; Índios Sul-Americanos 


\section{Introdução}

Conjuntamente com níveis elevados de pressão arterial, colesterol e glicose sanguíneos, a obesidade compõe o principal conjunto de fatores de risco associado à ocorrência de doenças crônicas não transmissíveis, que resultam em $60 \%$ das mortes no mundo a cada ano 1 . O excesso de peso e a obesidade são responsáveis por $44 \%$ e $23 \%$ da carga de doença devido ao diabetes mellitus e às doenças cardiovasculares, respectivamente, $\mathrm{e}$ entre $7 \%$ e $41 \%$ devido a certos tipos de câncer ${ }^{2}$.

Inquéritos nutricionais e de saúde realizados no Brasil nas últimas quatro décadas têm indicado um expressivo aumento nas prevalências de obesidade em adultos (de $8 \%$ para $16,9 \%$ em mulheres e de $2,8 \%$ para $12,4 \%$ em homens, entre 1974 e 2008) ${ }^{3}$. Tais informações, agregadas a outras sobre o consumo alimentar e perfil nutricional, evidenciam que a população brasileira vem experimentando franco processo de transição alimentar e nutricional, tal como tem sido observado em diversos outros países 4,5.

No Brasil, as informações sobre o processo da transição nutricional para os povos indígenas são limitadas ${ }^{6}$ quando comparadas às disponíveis para o restante da população ${ }^{7}$. A razão principal é que esse segmento populacional não tem sido sistematicamente incluído nos inquéritos de saúde e nutrição realizados no país ${ }^{8}$. No entanto, diversos estudos de caso em comunidades específicas têm apontado para a ocorrência de elevadas prevalências de excesso de peso e obesidade em adultos indígenas 9,10,11,12,13,14. Mais recentemente, os resultados do I Inquérito Nacional de Saúde e Nutrição dos Povos Indígenas, que teve por foco crianças menores de 5 anos e mulheres, mostraram que o excesso de peso e a obesidade atingem, respectivamente, $46,1 \%$ e $15,8 \%$ das mulheres de 14 a 49 anos 8 . Transformações socioeconômicas, em geral vinculadas a modificações nas atividades de subsistência e novas formas de trabalho, são consideradas importantes fatores relacionados a esse quadro, uma vez que se vinculam diretamente a padrões de alimentação e atividade física 15. Elevadas prevalências de obesidade, assim como das morbidades relacionadas, também têm sido descritas para povos indígenas em outros países nas Américas 16.

O objetivo deste estudo foi estimar as prevalências de excesso de peso e obesidade em adultos Xukuru do Ororubá em Pernambuco, Brasil, uma das maiores etnias indígenas do Nordeste, assim como avaliar os fatores socioeconômicos e demográficos associados a estes agravos. No conjunto das regiões brasileiras, a situação nutricional dos povos indígenas do Nordeste é a que tem sido menos investigada, apesar de seu contingente totalizar $25,5 \%$ do país 17 .

\section{Métodos}

Este trabalho, de corte transversal e base populacional, foi realizado na Terra Indígena (TI) Xukuru, localizada no Município de Pesqueira, Pernambuco. A TI Xukuru está situada na Mesorregião do Agreste Pernambucano, próximo à cidade de Pesqueira, distante aproximadamente $200 \mathrm{~km}$ da capital, Recife. Foram incluídos na investigação adultos com idades de 19,1 a 59 anos, residentes na $\mathrm{TI}$.

O contato dos Xukuru com a sociedade nacional envolvente data do período colonial e na segunda metade do século XIX chegaram a ser considerados extintos 18. Ao longo das últimas décadas, tem acontecido intenso processo de valorização de pertencimento étnico Xukuru. Em meados dos anos 1980, foi iniciado um movimento de retomada das terras tradicionais, o que resultou na homologação da TI Xukuru em 2001, com 27.555 hectares 19.

Os Xukuru do Orurubá constituem o maior contingente populacional indígena em Pernambuco (7.225 indivíduos), residindo em 25 aldeias distribuídas em três regiões geográficas da TI, conhecidas como Agreste, Serra e Ribeira do Ipojuca 20 . O Agreste é uma área montanhosa, com poucos terrenos disponíveis para a agricultura, considerada mais propícia para pecuária. A Serra é a região tida como de solos mais férteis da TI, onde estão localizados brejos de altitude, com nascentes de água. Finalmente, a região da Ribeira do Ipojuca é a mais árida 18.

\section{Plano amostral}

A amostra foi calculada para representar os 3.342 adultos Xukuru na faixa etária de 19,1 a 59 anos, assumindo-se uma prevalência de $50 \%$ para excesso de peso, precisão de $5 \%$ e intervalo de $95 \%$ de confiança. Desse modo, foi constituída por 414 indivíduos, já acrescidos de $20 \%$ para compensar eventuais perdas e recusas. Uma vez que este trabalho faz parte de uma investigação mais ampla sobre o perfil nutricional e de saúde dos Xukuru, que incluiu a avaliação da situação nutricional e de saúde bucal de crianças, adolescentes e idosos, foram amostrados os residentes de 1/3 dos domicílios na TI. Não obstante, a totalidade dos 1.091 adultos residentes nos 623 domicílios foi convidada a participar da pesquisa. A seleção dos domicílios ocorreu de forma aleatória sistemática. Todos eles foram numerados e a unidade amostral inicial selecionada por meio 
de sorteio entre 1 e k, em que k é o número total de domicílios. A cada três domicílios, o terceiro era selecionado.

\section{Coleta de dados}

A pesquisa de campo foi realizada no primeiro trimestre de 2010. As informações socioeconômicas e demográficas foram obtidas em janeiro e fevereiro, no âmbito do Diagnóstico Sociodemográfico Participativo dos Xukuru do Ororubá 20. Os entrevistadores eram agentes indígenas de saúde e de saneamento que trabalhavam na TI, tendo sido previamente treinados e continuamente supervisionados durante a pesquisa de campo. Os dados antropométricos e demais informações foram coletados no mês de março, ao longo de 27 dias corridos. Dezesseis entrevistadores, incluindo 12 estudantes de cursos de graduação da área da saúde, dois nutricionistas, um enfermeiro e um odontólogo, foram treinados para assegurar a qualidade das medidas antropométricas e o adequado preenchimento do instrumento de coleta de dados.

O peso corporal foi aferido utilizando-se balança digital portátil SECA (modelo 872, Hamburgo, Alemanha) e a estatura por meio de estadiômetro desmontável Alturexata (Belo Horizonte, Brasil).

\section{Variáveis de desfecho}

As variáveis analisadas neste trabalho incluem excesso de peso e obesidade, com base nos pontos de corte recomendados pela Organização Mundial da Saúde (OMS) 1, quais sejam, índice de massa corporal (IMC) $\geq 25,0 \mathrm{~kg} / \mathrm{m}^{2} \mathrm{e}$ $\geq 30,0 \mathrm{~kg} / \mathrm{m}^{2}$, respectivamente. O IMC foi calculado como a razão do peso corporal (em quilogramas) pelo quadrado da estatura (em metros). Foram também empregados os seguintes pontos de corte: baixo peso (IMC $<18,5 \mathrm{~kg} / \mathrm{m}^{2}$ ); eutrófico (IMC $\geq 18,5 \mathrm{e}<25,0 \mathrm{~kg} / \mathrm{m}^{2}$ ) e sobrepeso (IMC $\left.\geq 25,0 \mathrm{e}<30,0 \mathrm{~kg} / \mathrm{m}^{2}\right) 1$.

\section{Variáveis independentes}

Quanto às variáveis sociodemográficas, foram empregados os seguintes estratos: idade (19,129,9; 30,0-39,9; 40,0-49,9; 50,0-59,9 anos); sexo (masculino; feminino); região de moradia na TI (Ribeira, Agreste e Serra); situação conjugal (com ou sem companheiro); e escolaridade (anos de estudos concluídos com aprovação, nos estratos $0-2 ; 3-4 ; \geq 5$ anos).

No nível domiciliar, foram analisadas as seguintes variáveis: renda per capita; índice de status econômico (ISE); número de moradores no domicílio ( 1 -4; 5-8; $\geq 9$ moradores); número de moradores por cômodo ( $0-1 ; 2-3 ; \geq 4$ moradores); tipo de domicílio e tipo de saneamento.

A variável renda per capita foi definida baseando-se na soma dos valores monetários recebidos pelos moradores do domicílio com mais de 10 anos de idade (incluindo trabalho assalariado, pensões, aposentadoria, programas de transferência de renda e aquela gerada pela venda de produtos de artesanato e agropecuários), dividida pelo número de moradores. Com base no valor do salário mínimo à época da coleta de dados (R\$ 465,00), foram definidas três categorias $(<1 / 4$ salário mínimo; $\geq 1 / 4 \mathrm{e}<1 / 2$ salário mínimo; $\geq 1 / 2$ salário mínimo). Na modelagem estatística, ao se analisar padrões de interação entre variáveis, a renda per capita em salário mínimo foi considerada como variável contínua.

O ISE foi calculado a partir da soma (em Reais) dos valores dos seguintes bens de consumo, se presentes no domicílio, estimados com base nos preços no comércio regional: fogão a gás, geladeira, máquina de lavar roupa, ar condicionado, televisão, antena parabólica, vídeo cassete ou DVD, aparelho de som com CD, microcomputador, telefone celular e bicicleta. Foi calculada arazão $\mathrm{S}_{\mathrm{X}} / \max \left(\mathrm{S}_{\mathrm{X}}\right)$, na qual $\left(\mathrm{S}_{\mathrm{X}}\right)$ correspondeu à soma dos valores dos bens de um dado domicílio, e $\max \left(\mathrm{S}_{\mathrm{X}}\right)$ ao maior valor observado dentre todos os domicílios na TI. Esse indicador foi estratificado em três categorias (baixo, médio e alto), baseando-se nos terços da distribuição.

Foram definidas duas categorias de habitações. O tipo A incluiu aquelas que apresentavam parede de alvenaria, tijolo ou de madeira; piso de cerâmica ou cimento e telha de barro ou laje. As demais habitações foram classificadas como do tipo B, em geral apresentando materiais menos permanentes.

As condições de saneamento foram classificadas em três categorias: "adequada”, no caso de habitações com escoadouros ligados à rede geral ou fossa séptica, servidas de água proveniente de rede geral de abastecimento e lixo coletado direta ou indiretamente por serviço de limpeza pública; "semi-adequada", para aquelas com, pelo menos, um dos serviços de abastecimento de água, esgoto ou lixo classificado na categoria "adequada"; e "inadequada", aquelas com escoadouro ligado à fossa rudimentar, vala, rio, açude ou outro escoadouro, servidas de água proveniente de poço, nascente ou outra forma e com lixo queimado, enterrado ou jogado em terreno baldio.

\section{Análise de dados}

Inicialmente, foram calculadas as prevalências do excesso de peso e obesidade para as catego- 
rias de cada variável independente. Partindo do pressuposto de que o excesso de peso ou obesidade em um indivíduo estaria correlacionado ao perfil nutricional dos demais corresidentes, foi ajustado um modelo logístico multinível, com as características dos indivíduos no primeiro nível e aquelas do domicílio no segundo.

A análise dos efeitos das variáveis foi realizada em três etapas. Na primeira, modelos de regressão logística simples foram ajustados para cada uma das variáveis, selecionando-se aquelas estatisticamente significativas no nível de $20 \%$. A modelagem não incluiu os indivíduos que apresentaram IMC $<18,5 \mathrm{~kg} / \mathrm{m}^{2}$. Na segunda, um modelo foi ajustado por inclusão, passo a passo, das variáveis estatisticamente significativas ao nível de 5\%. Após a inclusão simultânea de todos os efeitos principais, foram testadas as interações plausíveis. Finalmente, na terceira e última etapa, foram incluídos os efeitos aleatórios para os domicílios.

Os modelos foram comparados com base no critério de informação de Akaike (AIC), de modo a considerar a qualidade do ajuste e a complexidade da modelagem. A associação e significância estatística de cada uma das variáveis independentes com o excesso de peso e a obesidade foram calculadas com base nas estimativas das razões de chances (RC).

\section{Ética}

O trabalho foi aprovado pelo Comitê de Ética em Pesquisa do Centro de Pesquisas Aggeu Magalhães, Fundação Oswaldo Cruz e pela Comissão Nacional de Ética em Pesquisa (CONEP, parecer no 592/09). Os investigados foram informados quanto à participação voluntária, sendo esclarecidos quanto aos objetivos e etapas. O Termo de Consentimento Livre e Esclarecido foi assinado pelo cacique da TI Xukuru e pelas lideranças de cada uma das 25 aldeias.

\section{Resultados}

Foram investigados 361 homens (45,8\%) e 433 mulheres $(54,2 \%)$. As perdas envolveram indivíduos que não foram incluídos por estarem ausentes de seus domicílios mesmo após três visitas por parte da equipe de entrevistadores ( $\mathrm{n}=162 ; 14,8 \%$ ); recusas quanto a participar do estudo ( $\mathrm{n}=32 ; 2,9 \%$ ); ou, na fase de análise dos dados, indivíduos para os quais não havia informações acerca de variáveis socioeconômicas e demográficas ( $\mathrm{n}=81 ; 7,4 \%$ ). Mulheres gestantes, que não fizeram parte do estudo, compunham $1,8 \%(n=20)$ dos sujeitos residentes nos domi- cílios selecionados. Desse modo, a amostra final foi composta por 794 indivíduos, correspondente a $192 \%$ daquela originalmente calculada. A amostra final expressivamente maior do que a necessária garantiu a representatividade do plano amostral, com precisão de $4 \%$ e intervalo de 99\% de confiança.

A Tabela 1 apresenta a distribuição dos indivíduos segundo as características socioeconômicas e demográficas e de estado nutricional. Predominaram pessoas na faixa etária de 19,1 a 29,9 anos, vivendo com companheiro, moradores da região da Serra e com menos de três anos de escolaridade formal. Para as variáveis domiciliares, o perfil mais comum foi aquele de pessoas com renda per capita $<1 / 4$ salário mínimo, residentes em domicílios do "tipo A", com saneamento básico "inadequado" e ISE "baixo". A maioria dos domicílios era habitada por 1 a 4 moradores e apresentava de 0-1 morador por cômodo. Foram observados perfis semelhantes para homens e mulheres.

Quanto ao estado nutricional, 2,6\% dos indivíduos apresentaram baixo peso, e o excesso de peso foi observado em $44,1 \%$ dos homens e $52,2 \%$ das mulheres. Quanto à obesidade, as prevalências foram de $7,5 \%$ e $21 \%$, respectivamente.

Considerando-se ambos os sexos, observouse que o IMC de um indivíduo selecionado aleatoriamente aumentava com o incremento do IMC médio dos demais moradores adultos do domicílio (Figura 1). Essa relação foi aproximadamente linear até $30,0 \mathrm{~kg} / \mathrm{m}^{2}$. Acima desse valor, não se verificou um padrão consistente. Tais achados reforçaram o pressuposto da ocorrência de correlação entre os desfechos de excesso de peso e obesidade para habitantes de um mesmo domicílio e justificou a utilização do modelo logístico multinível.

As prevalências dos agravos investigados e as razões de chance e seus respectivos intervalos de confiança são apresentados na Tabela 2 . $\mathrm{O}$ excesso de peso foi mais frequente em mulheres, em indivíduos com idades acima de 29,9 anos, vivendo com companheiro, com menos de 3 anos de escolaridade, com renda per capita $<1 / 2$ salário mínimo, com ISE médio e residentes em domicílio com saneamento básico "semi-adequado”. O padrão de distribuição da obesidade foi semelhante àquele observado para excesso de peso para as variáveis sexo, faixa etária, situação conjugal, escolaridade, ISE e condição do domicílio. As prevalências de ambos os agravos foram menores para indivíduos que residiam em domicílios com mais de $\geq 9$ moradores, assim como para aqueles em domicílios com $\geq 4$ moradores por cômodo. Os habitantes da região do Agreste apresentaram as maiores prevalências 
Tabela 1

Distribuição dos indivíduos Xukuru do Ororubá segundo as variáveis socioeconômicas e demográficas, de acordo com o sexo.

Pernambuco, Brasil, 2010.

\begin{tabular}{|c|c|c|c|c|c|c|}
\hline \multirow[t]{2}{*}{ Variáveis } & \multicolumn{2}{|c|}{ Homens } & \multicolumn{2}{|c|}{ Mulheres } & \multicolumn{2}{|c|}{ Total } \\
\hline & $\mathrm{n}$ & $\%$ & $\mathrm{n}$ & $\%$ & $\mathrm{n}$ & $\%$ \\
\hline \multicolumn{7}{|l|}{ Faixa etária (anos) } \\
\hline $19,1-29,9$ & 120 & 33,2 & 139 & 32,1 & 259 & 32,6 \\
\hline $30,0-39,9$ & 106 & 29,4 & 127 & 29,3 & 233 & 29,3 \\
\hline $40,0-49,9$ & 77 & 21,3 & 86 & 19,9 & 163 & 20,5 \\
\hline $50,0-59,9$ & 58 & 16,1 & 81 & 18,7 & 139 & 17,5 \\
\hline \multicolumn{7}{|l|}{ Situação conjugal } \\
\hline Com companheiro & 255 & 70,6 & 323 & 74,6 & 578 & 72,8 \\
\hline Sem companheiro & 106 & 29,4 & 110 & 25,4 & 216 & 27,2 \\
\hline \multicolumn{7}{|c|}{ Escolaridade (anos de estudo) } \\
\hline $0-2$ & 156 & 43,2 & 156 & 36,0 & 312 & 39,3 \\
\hline $3-4$ & 96 & 26,6 & 125 & 28,9 & 221 & 27,8 \\
\hline$\geq 5$ & 109 & 30,2 & 152 & 35,1 & 261 & 32,9 \\
\hline \multicolumn{7}{|l|}{ Estado nutricional } \\
\hline Baixo peso & 7 & 1,9 & 14 & 3,2 & 21 & 2,6 \\
\hline Eutrofia & 195 & 54,0 & 193 & 44,6 & 388 & 48,9 \\
\hline Sobrepeso & 132 & 36,6 & 135 & 31,2 & 267 & 33,6 \\
\hline Obesidade & 27 & 7,5 & 91 & 21,0 & 118 & 14,9 \\
\hline \multicolumn{7}{|l|}{ Região socioambiental } \\
\hline Ribeira & 99 & 27,4 & 121 & 27,9 & 220 & 27,7 \\
\hline Agreste & 114 & 31,6 & 140 & 32,3 & 254 & 32,0 \\
\hline Serra & 148 & 41,0 & 172 & 39,7 & 320 & 40,3 \\
\hline \multicolumn{7}{|c|}{ Renda per capita (salário mínimo) } \\
\hline$<1 / 4$ & 175 & 48,5 & 221 & 51,0 & 396 & 49,9 \\
\hline$\geq 1 / 4$ e $<1 / 2$ & 99 & 27,4 & 113 & 26,1 & 212 & 26,7 \\
\hline$\geq 1 / 2$ & 87 & 24,1 & 99 & 22,9 & 186 & 23,4 \\
\hline \multicolumn{7}{|c|}{ Índice de status econômico (ISE) } \\
\hline Baixo & 162 & 44,9 & 205 & 47,3 & 367 & 46,2 \\
\hline Médio & 84 & 23,3 & 109 & 25,2 & 193 & 24,3 \\
\hline Alto & 115 & 31,9 & 119 & 27,5 & 234 & 29,5 \\
\hline \multirow{2}{*}{\multicolumn{7}{|c|}{$\begin{array}{l}\text { Número de moradores no } \\
\text { domićlio }\end{array}$}} \\
\hline & & & & & & \\
\hline $1-4$ & 208 & 57,6 & 245 & 56,6 & 453 & 57,1 \\
\hline $5-8$ & 119 & 33,0 & 153 & 35,3 & 272 & 34,3 \\
\hline$\geq 9$ & 34 & 9,4 & 35 & 8,1 & 69 & 8,7 \\
\hline \multicolumn{7}{|c|}{ Número de moradores por } \\
\hline \multicolumn{7}{|l|}{ cômodos } \\
\hline $0-1$ & 255 & 70,6 & 297 & 68,6 & 552 & 69,5 \\
\hline $2-3$ & 46 & 12,7 & 65 & 15,0 & 111 & 14,0 \\
\hline$\geq 4$ & 60 & 16,6 & 71 & 16,4 & 131 & 16,5 \\
\hline \multicolumn{7}{|c|}{ Caracterização da habitação } \\
\hline Tipo A & 319 & 88,4 & 381 & 88,0 & 700 & 88,2 \\
\hline Tipo B & 42 & 11,6 & 52 & 12,0 & 94 & 11,8 \\
\hline \multicolumn{7}{|l|}{ Tipo de saneamento } \\
\hline Adequado & 6 & 1,7 & 6 & 1,4 & 12 & 1,5 \\
\hline Semi-adequado & 153 & 42,4 & 201 & 46,4 & 354 & 44,6 \\
\hline Inadequado & 202 & 56,0 & 226 & 52,2 & 428 & 53,9 \\
\hline
\end{tabular}


Relação entre o índice de massa corporal (IMC) de indivíduos adultos Xukuru do Ororubá selecionados ao acaso dentro de um domicílio (eixo y) e o IMC médio dos co-habitantes do mesmo domicílio (eixo x). Pernambuco, Brasil, 2010.

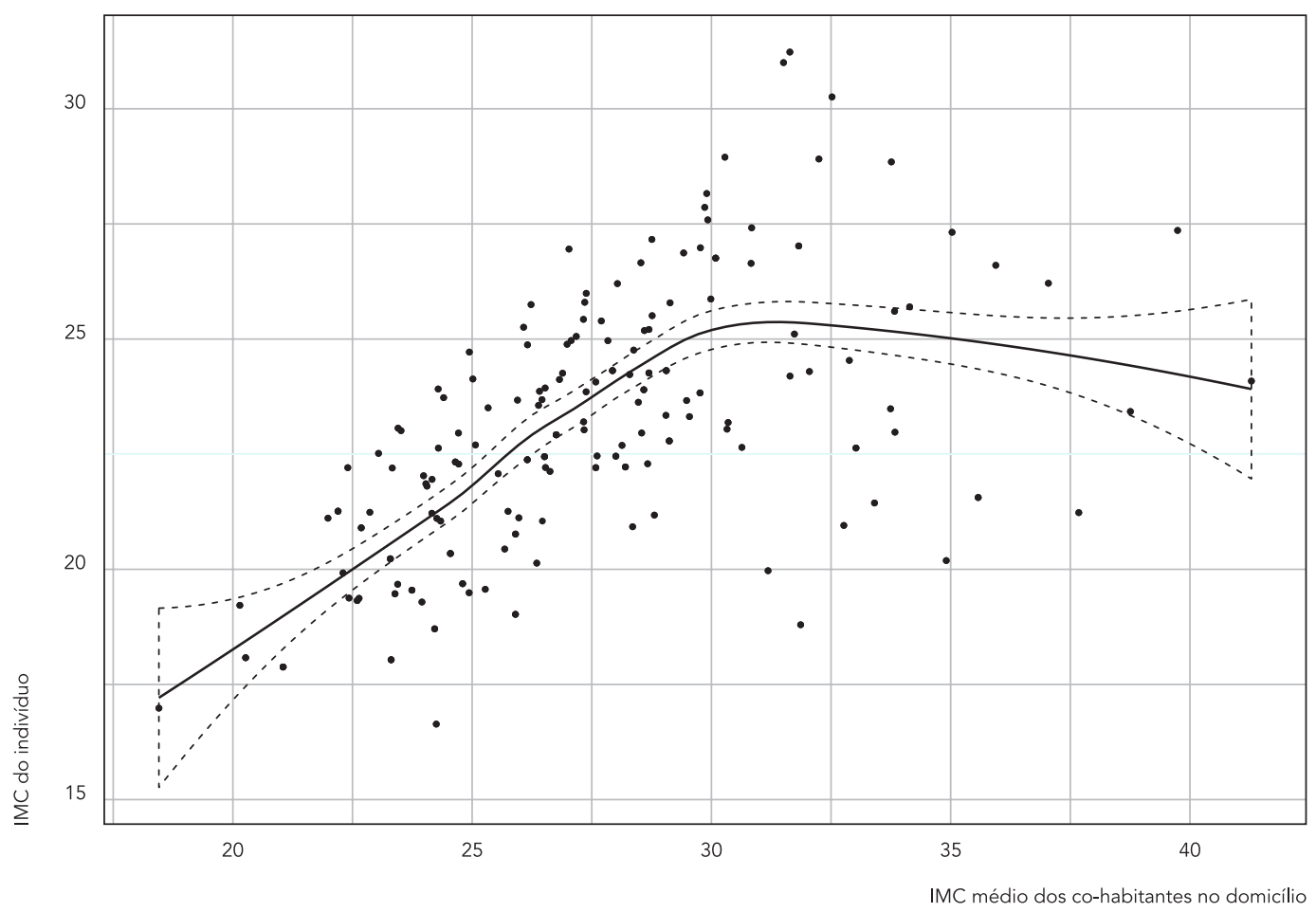

de excesso de peso e as menores de obesidade (Tabela 2).

Na Tabela 3, são apresentados os resultados do modelo logístico multinível. As variáveis idade e sexo estiveram associadas ao excesso de peso. As mulheres foram mais propensas a apresentar excesso de peso e obesidade. A chance de apresentar excesso de peso aumentou com a idade, enquanto que para as demais variáveis independentes não foram observadas associações estatisticamente significativas. Não obstante, tais variáveis foram mantidas no modelo para fins de comparação com aquele referente à obesidade.

Em relação à obesidade, além das variáveis idade e sexo, ISE e renda per capita também foram estatisticamente significativas. Se comparadas aos homens, as mulheres mostraram chance mais acentuada de apresentar obesidade. A variável renda per capita, incluída no modelo final de forma contínua em salários mínimos, foi associada à obesidade e apresentou interação com sexo. A Figura 2 mostra que, para os homens, o aumento da renda resultou em uma maior pro- babilidade de ser obeso, e para as mulheres tal probabilidade permanece constante, independentemente do valor da renda.

\section{Discussão}

Os resultados do presente estudo, derivados de uma amostra representativa de adultos do povo Xukuru do Ororubá, revelam elevadas prevalências de sobrepeso e obesidade $(33,5 \%$ e $14,8 \%$, respectivamente, para os sexos combinados) e reduzida frequência de baixo peso $(2,9 \%$, sexos combinados). Tais achados estão alinhados com aqueles de outros estudos sobre povos indígenas no Brasil e no mundo, que têm apontado para a emergência de excesso de peso associado a contextos de acelerados processos de transição nutricional 9,10,11,12,13,14,21,22,23.

De uma maneira geral, o perfil de saúde de povos indígenas tem sido descrito como apresentando diferenças importantes em relação àquele de não indígenas em diversos países 16 . 
Tabela 2

Prevalência de excesso de peso e obesidade e razão de chance bruta, segundo variáveis socioeconômicas e demográficas para adultos Xukuru do Ororubá Pernambuco, Brasil, 2010.

\begin{tabular}{|c|c|c|c|c|c|c|}
\hline \multirow[t]{2}{*}{ Variáveis } & \multicolumn{3}{|c|}{ Excesso de peso (IMC $\left.\geq 25 \mathrm{~kg} / \mathrm{m}^{2}\right)$} & \multicolumn{3}{|c|}{ Obesidade (IMC $\geq 30 \mathrm{~kg} / \mathrm{m}^{2}$ ) } \\
\hline & $\mathbf{n}$ & Prevalência (\%) & RC (IC95\%) & $\mathbf{n}$ & Prevalência (\%) & RC (IC95\%) \\
\hline \multicolumn{7}{|l|}{ Sexo } \\
\hline Masculino & 159 & 44,9 & 1,00 & 27 & 7,6 & 1,00 \\
\hline Feminino & 226 & 53,9 & $1,44(1,08-1,91)$ & 91 & 21,7 & $3,42(2,13-5,49)$ \\
\hline \multicolumn{7}{|l|}{ Região } \\
\hline Ribeira & 108 & 50,5 & 1,00 & 34 & 15,9 & 1,00 \\
\hline Agreste & 129 & 51,8 & $1,06(0,73-1,52)$ & 36 & 14,5 & $0,94(0,55-1,60)$ \\
\hline Serra & 148 & 47,7 & $0,9(0,63-1,27)$ & 48 & 15,5 & $0,92(0,56-1,52)$ \\
\hline \multicolumn{7}{|l|}{ Faixa etária (anos) } \\
\hline $19,1-29,9$ & 96 & 38,6 & 1,00 & 21 & 8,4 & 1,00 \\
\hline $30,0-39,9$ & 124 & 54,1 & $1,88(1,31-2,71)$ & 41 & 17,9 & $2,84(1,59-5,09)$ \\
\hline $40,0-49,9$ & 87 & 54,7 & $1,93(1,29-2,88)$ & 26 & 16,4 & $2,59(1,37-4,92)$ \\
\hline $50,0-59,9$ & 78 & 57,4 & $2,14(1,4-3,28)$ & 30 & 22,1 & $3,77(2,00-7,11)$ \\
\hline \multicolumn{7}{|l|}{ Situação conjugal } \\
\hline Com companheiro & 297 & 52,4 & 1,00 & 96 & 16,9 & 1,00 \\
\hline Sem companheiro & 88 & 42,7 & $0,68(0,49-0,94)$ & 22 & 10,7 & $0,53(0,32-0,88)$ \\
\hline \multicolumn{7}{|c|}{ Escolaridade (anos de estudo) } \\
\hline $0-2$ & 164 & 53,9 & 1,00 & 50 & 16,4 & 1,00 \\
\hline $3-4$ & 107 & 49,5 & $0,84(0,59-1,19)$ & 31 & 14,4 & $0,79(0,47-1,32)$ \\
\hline$\geq 5$ & 114 & 45,1 & $0,7(0,50-0,98)$ & 37 & 14,6 & $0,75(0,46-1,21)$ \\
\hline \multicolumn{7}{|c|}{ Renda per capita (salário mínimo) } \\
\hline$<1 / 4$ & 187 & 49,1 & 1,00 & 47 & 12,3 & 1,00 \\
\hline$\geq 1 / 4$ e $<1 / 2$ & 112 & 53,3 & $1,19(0,85-1,66)$ & 38 & 18,1 & $1,61(0,98-2,63)$ \\
\hline$\geq 1 / 2$ & 86 & 47,3 & $0,93(0,65-1,32)$ & 33 & 18,1 & $1,43(0,86-2,37)$ \\
\hline \multicolumn{7}{|c|}{ Índice de status econômico (ISE) } \\
\hline Baixo & 101 & 45,3 & 1,00 & 22 & 9,9 & 1,00 \\
\hline Médio & 190 & 52,8 & $1,35(0,97-1,89)$ & 68 & 18,9 & $2,24(1,31-3,81)$ \\
\hline Alto & 94 & 49,5 & $1,18(0,80-1,74)$ & 28 & 14,7 & $1,63(0,88-3,03)$ \\
\hline \multicolumn{7}{|c|}{ Número de moradores no domicílio } \\
\hline $1-4$ & 227 & 51,1 & 1,00 & 68 & 15,3 & 1,00 \\
\hline $5-8$ & 130 & 48,9 & $0,91(0,67-1,24)$ & 43 & 16,2 & $1,00(0,65-1,56)$ \\
\hline$\geq 9$ & 28 & 44,4 & $0,76(0,45-1,30)$ & 7 & 11,1 & $0,62(0,26-1,46)$ \\
\hline \multicolumn{7}{|c|}{ Número de moradores por cômodo } \\
\hline $0-1$ & 287 & 53,1 & 1,00 & 85 & 15,7 & 1,00 \\
\hline $2-3$ & 55 & 51,9 & $0,95(0,63-1,44)$ & 17 & 16,0 & $0,97(0,53-1,77)$ \\
\hline$\geq 4$ & 43 & 33,9 & $0,45(0,30-0,68)$ & 16 & 12,6 & $0,57(0,31-1,02)$ \\
\hline \multicolumn{7}{|c|}{ Caracterização da habitação } \\
\hline Tipo B & 41 & 46,1 & 1,00 & 13 & 14,6 & 1,00 \\
\hline Tipo A & 344 & 50,3 & $1,18(0,76-1,84)$ & 105 & 15,4 & $1,16(0,61-2,23)$ \\
\hline \multicolumn{7}{|l|}{ Tipo de saneamento } \\
\hline Adequado & 5 & 45,5 & 1,00 & 2 & 18,2 & 1,00 \\
\hline Semi-adequado & 185 & 53,0 & $1,35(0,41-4,52)$ & 50 & 14,3 & $0,91(0,18-4,67)$ \\
\hline Inadequado & 195 & 47,2 & $1,07(0,32-3,57)$ & 66 & 16,0 & $0,90(0,18-4,59)$ \\
\hline
\end{tabular}

IC95\%: intervalo de 95\% de confiança; IMC: índice de massa corporal; RC: razão de chances. 
Tabela 3

Modelo ajustado de regressão logística multinível para excesso de peso e obesidade em adultos Xukuru do Ororubá. Pernambuco, Brasil, 2010.

\begin{tabular}{|c|c|c|c|c|}
\hline \multirow[t]{2}{*}{ Variáveis } & \multicolumn{2}{|c|}{ Excesso de peso $\left(\mathrm{IMC} \geq 25 \mathrm{~kg} / \mathrm{m}^{2}\right)$} & \multicolumn{2}{|c|}{ Obesidade (IMC $\geq 30 \mathrm{~kg} / \mathrm{m}^{2}$ ) } \\
\hline & RC (IC95\%) & Valor de p & RC (IC95\%) & Valor de $p$ \\
\hline \multicolumn{5}{|l|}{ Sexo } \\
\hline Masculino & 1,00 & & 1,00 & \\
\hline Feminino & $1,72(1,10-2,69)$ & 0,02 & $8,40(3,52-20,04)$ & $<0,01$ \\
\hline \multicolumn{5}{|l|}{ Faixa etária (anos) } \\
\hline $19,1-29,9$ & 1,00 & & 1,00 & \\
\hline $30,0-39,9$ & $1,88(1,29-2,74)$ & $<0,01$ & $3,31(1,68-6,56)$ & $<0,01$ \\
\hline $40,0-49,9$ & $2,02(1,34-3,07)$ & $<0,01$ & $3,33(1,59-6,99)$ & 0,01 \\
\hline $50,0-59,9$ & $2,16(1,38-3,37)$ & $<0,01$ & $4,30(2,02-9,17)$ & 0,01 \\
\hline \multicolumn{5}{|l|}{ Índice de status econômico (ISE) } \\
\hline Baixo & 1,00 & & 1,00 & \\
\hline Médio & $1,27(0,89-1,83)$ & 0,19 & $2,15(1,11-4,17)$ & 0,02 \\
\hline Alto & $1,11(0,72-1,70)$ & 0,63 & $1,55(0,71-3,38)$ & 0,27 \\
\hline Renda per capita & $1,52(0,70-3,33)$ & 0,29 & $5,34(1,30-21,86)$ & 0,02 \\
\hline \multicolumn{5}{|l|}{ Sexo x renda per capita } \\
\hline Masculino $x$ renda per capita & 1,00 & & 1,00 & \\
\hline Feminino $x$ renda per capita & $0,58(0,21-1,59)$ & 0,08 & $0,15(0,03-0,81)$ & 0,03 \\
\hline
\end{tabular}

IC95\%: intervalo de 95\% de confiança; IMC: índice de massa corporal; RC: razão de chances.

Independentemente do local onde habitam no mundo, os indígenas tendem a apresentar os piores indicadores de saúde, incluindo menor esperança de vida ao nascer e níveis mais elevados de mortalidade infantil, materna e geral 16 . No Brasil, diversas pesquisas que compararam os perfis de saúde de indígenas e não indígenas apontaram para a ocorrência de marcadas iniquidades. Nesse âmbito, podem ser citadas as análises sobre déficit de crescimento e anemia em menores de cinco anos, cujas prevalências são, respectivamente, quatro e duas vezes maiores quando comparadas àquelas de crianças não indígenas 24,25 .

Prevalências de obesidade superiores às reportadas no contexto nacional já foram descritas em diversas investigações sobre povos indígenas no Brasil. Estudos realizados em diferentes comunidades Xavante no Mato Grosso reportaram frequências próximas de $22 \%$ e $40 \%$ em homens e mulheres, respectivamente 11,12. Em indígenas das etnias Teréna, Guarani e Kaiowá, no Mato Grosso do Sul, 58,2\% dos homens e $66,9 \%$ das mulheres apresentaram excesso de peso, enquanto que a proporção de mulheres obesas $(30,8 \%)$ foi praticamente o dobro daquela observada em homens $(14,2 \%){ }^{14}$. Portanto, a ocorrência de obesidade nos Xukuru esteve menos frequente do que a observada nessas outras investigações 11,12,14.
A prevalência de obesidade nas mulheres Xukuru $(21,1 \%)$ é superior àquela reportada no mais recente estudo de abrangência nacional acerca da população brasileira (16,9\%) 3 . No contexto do Estado de Pernambuco, Pinho et al. 26, em investigação realizada com amostra representativa da população com mais de 25 anos, reportaram taxas de obesidade de $12,8 \%$ para os homens e de $20,7 \%$ para as mulheres. Portanto, as prevalências de obesidade para as mulheres Xukuru foram semelhantes àquelas observadas no âmbito estadual, e os homens indígenas apresentaram prevalências mais baixas. Quando os resultados são confrontados com os obtidos no $I$ Inquérito Nacional de Saúde e Nutrição dos Povos Indígenas 8 , observa-se que as mulheres Xukuru apresentaram prevalências mais elevadas de obesidade, que foi de $15,8 \%$ (total) e 13,5\% (Nordeste) segundo o inquérito.

Estudo realizado no Estado do Acre 27, que apontou elevadas prevalências de excesso de peso e obesidade em indivíduos adultos de ambos os sexos, evidenciou que, para os homens, o maior risco de excesso de peso foi observado naqueles que pertenciam aos maiores estratos de renda. Nos homens Xukuru foi encontrada uma associação positiva entre o aumento da renda e a maior probabilidade de obesidade.

Em termos de magnitude, as prevalências de excesso de peso e obesidade entre homens 
Figura 2

Gráficos de efeito entre a probabilidade (0-1) de ser obeso e faixa etária, e a probabilidade de ser obeso e a interação entre sexo e renda per capita, para adultos Xukuru do Ororubá. Pernambuco, Brasil, 2010.

2a) Probabilidade de ser obeso e faixa etária.

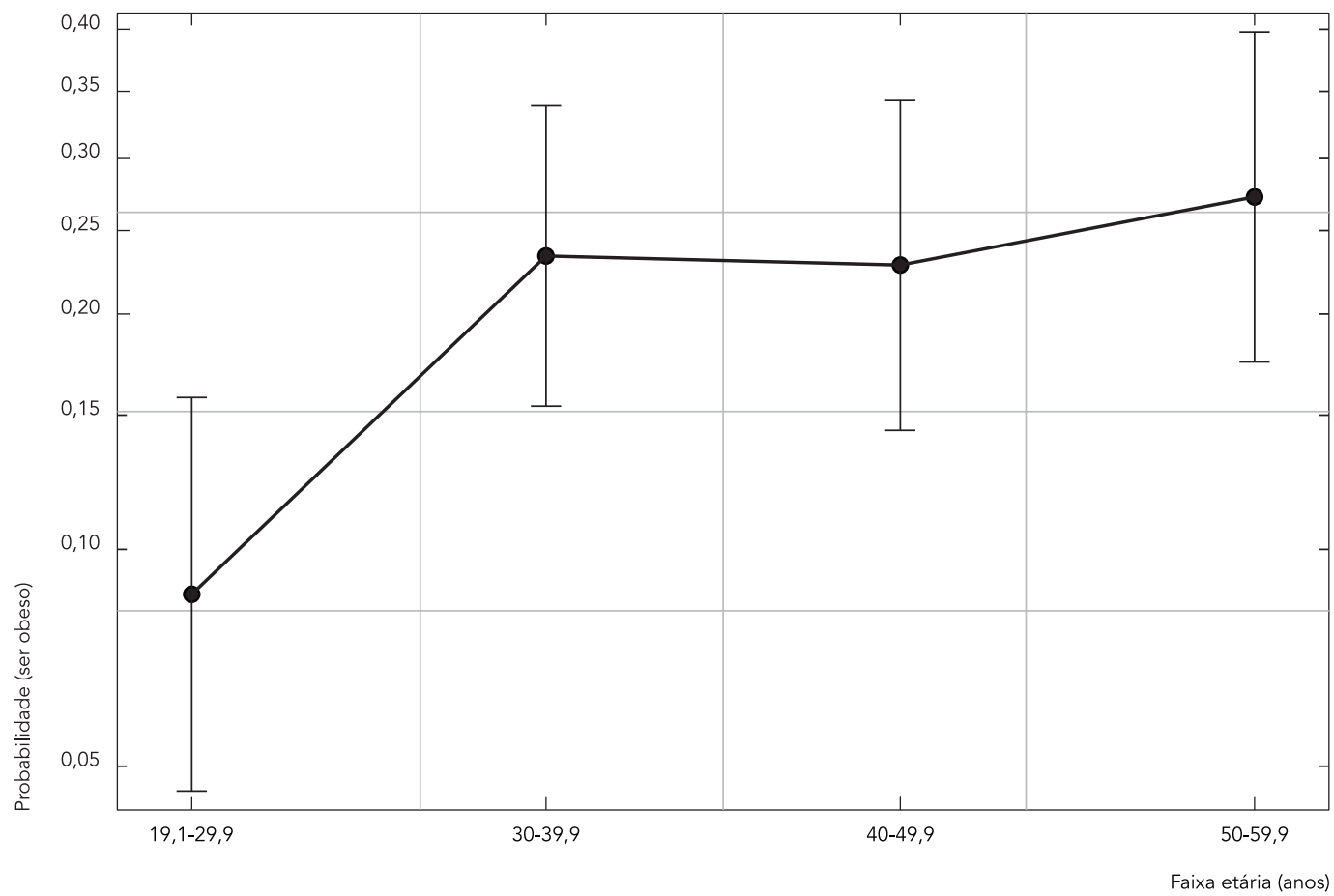

2b)Probabilidade de ser obeso e a interação entre sexo e renda per capita.

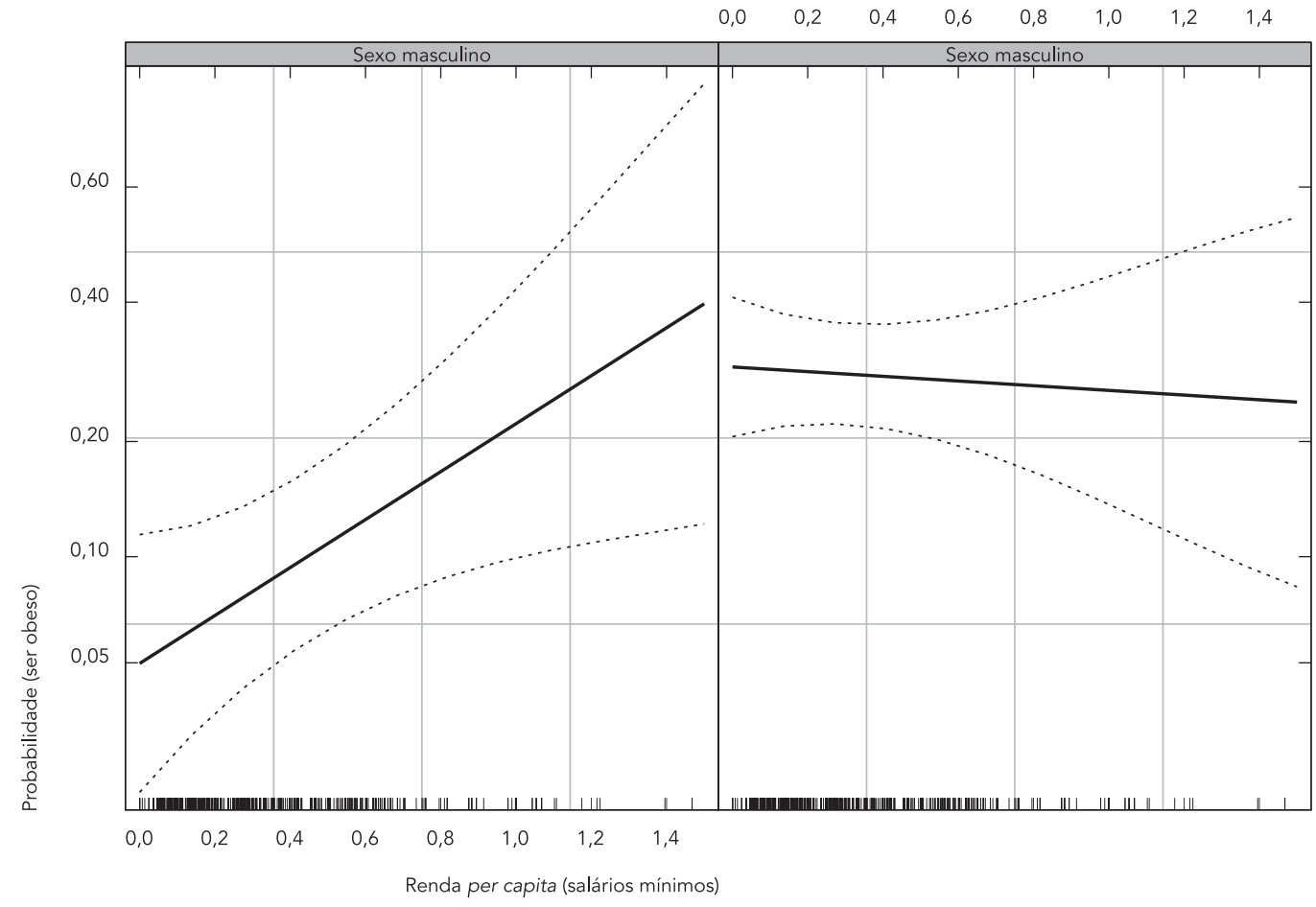


Xukuru divergiram dos resultados do último estudo de âmbito nacional (Pesquisa de Orçamentos Familiares - POF 2008/2009), se considerado o Brasil como um todo ${ }^{3}$. A razão da prevalência de obesidade ao se comparar homens não indígenas e Xukuru foi de 1,6. No entanto, quando são considerados apenas os resultados de homens residentes na área rural do Brasil e na rural do Nordeste, observam-se diminuições das razões (1,5 e 0,7, respectivamente). Outros trabalhos realizados com adultos residentes em áreas rurais do Brasil apontaram para taxas de excesso de peso e obesidade mais baixas nos homens não indígenas 25,28,29.

Pinho et al. 26 afirmam que a menor prevalência de excesso de peso e obesidade em homens que viviam em área rural de Pernambuco pode ser atribuída a fatores alimentares e ao nível de atividade física. Isso porque, em contextos rurais, pressupõe-se um maior gasto energético em atividades laborais e um menor acesso a alimentos industrializados, de alta densidade calórica e de gorduras. Possivelmente esses também são fatores relacionados às prevalências mais baixas de obesidade entre os homens Xukuru, sendo estas ainda inferiores, inclusive, àquelas observadas em homens não indígenas residentes na área rural de Pernambuco.

Foi observada uma relação direta entre a probabilidade de excesso de peso e obesidade e a idade dos adultos Xukuru. Para ambos os sexos, a diferença nas taxas de prevalência de obesidade, entre os grupos de menor e maior idade, foi de aproximadamente três vezes. A associação positiva entre a idade e o excesso de peso tem sido frequentemente relatada na literatura científica e, na maioria dos casos, vinculada ao sexo feminino, sendo esta associação atribuída, em grande parte, à diminuição da taxa metabólica basal que acompanha o avanço da idade e a redução da prática de atividades físicas 30 . Expressiva parte dos estudos com foco no estado nutricional de adultos no Brasil, quer seja incluindo indígenas ou não, engloba também os indivíduos com mais de 60 anos de idade. No caso Xukuru, esse segmento da população será alvo de outra publicação, uma vez que a falta de consenso sobre os pontos de corte de IMC para a definição de excesso de peso, obesidade e baixo peso, demanda detalhamento que vai além dos objetivos do presente trabalho.

Foi encontrada uma associação positiva entre probabilidade de obesidade e renda entre os homens Xukuru, padrão similar ao reportado para homens adultos residentes em Pernambuco 26. Esses resultados estão alinhados com outros que indicam que tal associação é mais predominante para o sexo masculino ${ }^{31}$. No Brasil, desde
1989, observa-se o deslocamento da ocorrência de maiores prevalências de obesidade para o segmento de menor nível socioeconômico. Nas últimas décadas, no tocante à dimensão socioeconômica, as diferenças no perfil nutricional diminuíram em homens e mulheres, ainda que de forma mais acentuada para o sexo feminino 4 .

Os determinantes dos agravos nutricionais em indígenas estão relacionados a inúmeras transformações de ordem demográfica, socioeconômica, cultural, ambiental e epidemiológica $8,15,16$. Em conjunto com novos padrões de consumo alimentar estabelecidos nas últimas décadas, que incluem a incorporação de produtos industrializados de elevado teor energético, diversos estudos realizados em comunidades indígenas apontam para a redução dos níveis de atividade física, inclusive no contexto das atividades laborais 10,11,12,21. Lourenço et al. 10, com base em investigação realizada com os Suruí, Rondônia observaram estreita associação entre as elevadas prevalências de excesso de peso e obesidade em homens e mulheres com diferentes gradientes socioeconômicos presentes na comunidade, associados a novos hábitos alimentares e padrões de atividade física. No povo Xavante, Mato Grosso, também foi encontrada associação positiva entre obesidade e status socioeconômico ${ }^{11}$. Tal como em outros planos de determinação da saúde indígena, características históricas, socioeconômicas e de infraestrutura exercem influência na escolha e disponibilidade de alimentos, o que os torna centrais na ocorrência do sobrepeso e obesidade entre esses povos.

Quanto às limitações deste estudo, para uma melhor compreensão da epidemiologia do excesso de peso e da obesidade entre os Xukuru seria fundamental a inclusão de informações acerca dos padrões de consumo alimentar e atividade física. Outro ponto diz respeito ao uso do IMC para inferir quanto ao excesso de gordura corporal. Isso porque, por meio de tal indicador, não é possível distinguir, em particular no caso de valores elevados, tecido gorduroso em relação ao muscular. No entanto, devido à sua elevada correlação com o excesso de gordura corporal total, o IMC tem sido recomendado para uso em estudos populacionais 32 , de modo que vem sendo amplamente utilizado em pesquisas envolvendo povos indígenas 15. Gugelmin \& Santos 33 observaram alta correlação do IMC com as medidas antropométricas indicativas de acúmulo de gordura corporal entre os Xavante.

Em síntese, observou-se que excesso de peso e obesidade são altamente prevalentes entre os adultos Xukuru. Sexo, idade e status econômico estiveram associados à obesidade, assim como a interação sexo masculino e renda per capita. Foi 
também verificada associação positiva quanto à ocorrência de sobrepeso em moradores de um mesmo domicílio. Em plano mais geral, os resultados deste estudo, assim como diversos outros realizados no país nos últimos anos, evidenciam que o excesso de peso e a obesidade se destacam no cenário nutricional de muitos povos in- dígenas. Tal contexto deve ser levado em consideração nas políticas e ações de saúde pública. Ressalte-se que, até o momento, não existem no Brasil ações específicas no campo da saúde dos povos indígenas voltadas para o controle das doenças crônicas não transmissíveis, quer seja no nível local ou em um plano nacional mais amplo.

\section{Resumen}

Este estudio transversal tiene como objetivo describir la distribución del exceso de peso, obesidad y su asociación con variables demográficas y socioeconómicas. El estudio fue realizado entre 794 adultos indigenas, de 19 hasta 59 años de edad de los Xukuru do Orurubá, pueblo indígena cuyas tierras están ubicadas en el Municipio de Pesqueira, Pernambuco, Brasil. El análisis de la asociación entre variables de desenlace, exceso de peso (IMC > 24,99kg/m²), obesidad (> 29,99kg/m²) y las variables explicativas fue realizado mediante una regresión logística multinivel. Entre las mujeres, un 52\% estaban con exceso de peso y un $21 \%$ obesos, en el caso de los hombres. Por otro lado, la prevalencia fue de un $44,1 \%$ y un 7,5\%, respectivamente. Las variables sexo femenino y edad (> 30 años) se mostraron asociadas a la ocurrencia de ambas condiciones. El estatus socioeconómico y la interacción entre sexo masculino y renta per cápita elevada mostraron asociación con obesidad. Así como lo observado en otras poblaciones indígenas, los resultados sugieren que los Xukuru están atravesando un acelerado proceso de transición nutricional.

Obesidad; Índice de Masa Corporal; Encuestas Nutricionales; Indios Sudamericanos

\section{Colaboradores}

T. R. Fávaro participou da concepção do projeto, coordenou a coleta de dados, realizou a análise dos dados, interpretação dos resultados, redação do manuscrito e revisão final do texto. R. V. Santos participou da discussão dos resultados, redação e revisão do texto final. G. M. Cunha e I. C. Leite contribuíram com a análise dos dados, redação, revisão crítica do manuscrito e aprovação final da versão a ser publicada. C. E. A. Coimbra Jr. participou da discussão dos resultados, redação e revisão do texto final.

\section{Agradecimentos}

Agradecemos a população Xukuru do Ororubá pela recepção à equipe de pesquisa e aos pesquisadores do Centro de Pesquisas Aggeu Magalhães, Fundação Oswaldo Cruz (CpqAM/Fiocruz), em especial ao pesquisador André Monsteiro Costa. Esta pesquisa é derivada de projetos financiados pela FACEPE (processo no APQ-1351-4.00/08) e CNPq (processo no 475681/2008) e foi realizada durante a vigência bolsa de doutorado (CNPq) e pós-doutorado (Convênio Fiocruz/Capes - Brasil sem Miséria) concedida à primeira autora do artigo. 


\section{Referências}

1. World Health Organization. 2008-2013 action plan for the global strategy for the prevention and control of noncommunicable diseases. http://whqlib doc.who.int/publications/2009/9789241597418_ eng.pdf (acessado em 05/Mai/2013).

2. World Health Organization. Overweight and obesity. Geneva: World Health Organization; 2011. (Fact Sheet, 311).

3. Instituto Brasileiro de Geografia e Estatística. Pesquisa de Orçamentos Familiares 2008-2009: antropometria e estado nutricional de crianças, adolescentes e adultos no Brasil. Rio de Janeiro: Instituto Brasileiro de Geografia e Estatística; 2010.

4. Monteiro CA, Conde WL, Popkin BM. Income-specific trends in obesity in Brazil: 1975-2003. Am J Public Health 2007; 97:1808-12.

5. Moura EC, Claro RM. Estimates of obesity trends in Brazil, 2006-2009. Int J Public Health 2012; 57:127-33.

6. Santos RV, Coimbra Jr. CEA. Cenários e tendências da saúde e da epidemiologia dos povos indígenas no Brasil. In: Coimbra Jr. CEA, Santos RV, Escobar AL, organizadores. Epidemiologia e saúde dos povos indígenas no Brasil. Rio de Janeiro: Editora Fiocruz; 2003. p. 13-47.

7. Monteiro CA, Benício MHDA, Conde WL, Popkin BM. Shifting obesity trends in Brazil. Eur J Clin Nutr 2000; 54:342-6.

8. Coimbra Jr. CEA, Santos RV, Welch JR, Cardoso AM, Souza MC, Garnelo L, et al. The First National Survey of Indigenous People's Health and Nutrition in Brazil: rationale, methodology, and overview of results. BMC Public Health 2013;13:52.

9. Gimeno SGA, Rodrigues D, Pagliaro H, Cano EN, Lima EED, Baruzzi RG. Perfil metabólico e antropométrico de índios Aruák: Mehináku, Waurá e Yawalapití, Alto Xingu, Brasil Central, 2000/2002 . Cad Saúde Pública 2007; 23:1946-54.

10. Lourenço AE, Santos RV, Orellana JD, Coimbra Jr. CEA. Nutrition transition in Amazonia: obesity and socioeconomic change in the Suruí Indians from Brazil. Am J Hum Biol 2008; 20:564-71.

11. Welch JR, Ferreira AA, Santos RV, Gugelmin SA, Werneck G, Coimbra Jr. CEA. Nutrition transition, socioeconomic differentiation, and gender among adult Xavante Indians, Brazilian Amazon. Hum Ecol 2009; 37:13-26.

12. Gugelmin SA, Santos RV. Ecologia humana e antropometria nutricional de adultos Xavánte, Mato Grosso, Brasil. Cad Saúde Pública 2001; 17:313-22.

13. Salvo VLMA, Rodrigues D, Baruzzi RG, Pagliaro H, Gimeno SGA. Perfil metabólico e antropométrico dos Suyá: Parque Indígena do Xingu, Brasil Central. Rev Bras Epidemiol 2009; 12:458-68.

14. Oliveira GF, Oliveira TR, Rodrigues FF, Corrêa LF, Ikejiri AT, Casulari LA. Prevalence of diabetes mellitus and impaired glucose tolerance in indigenous people from Aldeia Jaguapiru, Brazil. Rev Panam Salud Pública 2011; 29:315-21.
15. Leite MS, Santos RV, Coimbra Jr. CEA, Gugelmin SA. Alimentação e nutrição dos povos indígenas no Brasil. In: Kac G, Sichieri R, Gigante DP, organizadores. Epidemiologia nutricional. Rio de Janeiro: Editora Fiocruz/Editora Atheneu; 2007. p. 503-17.

16. Gracey M, King M. Indigenous health. Part 1: determinants and disease patterns. Lancet 2009; 374:65-75.

17. Instituto Brasileiro de Geografia e Estatística. Os indígenas no Censo Demográfico de 2010: primeiras considerações. Rio de Janeiro: Instituto Brasileiro de Geografia e Estatística; 2012.

18. Silva E. História, memórias e identidade entre os Xukuru do Ororubá. Revista Tellus 2007; 12:89-102.

19. Neves RCM. Resistência e estratégias de mobilização entre os Xukuru. In: Athias R, organizador. Povos indígenas de Pernambuco: identidade, diversidade e conflito. Recife: Editora UFPE; 2007. p. 113-36.

20. Teixeira P, Costa AM. Xukuru de Ororubá: introdução à demografia de um povo indígena. In: Anais do XVIII Encontro Nacional de Estudos Populacionais. http://www.abep.nepo.unicamp.br/xviii/ anais/files/ST4[694]ABEP2012.pdf (acessado em 24/Set/2013).

21. Gimeno SG, Rodrigues D, Canó EN, Lima EE, Schaper M, Pagliaro H, et al. Cardiovascular risk factors among Brazilian Karib indigenous peoples: Upper Xingu, Central Brazil, 2000-3. J Epidemiol Community Health 2009; 63:299-304.

22. Orden AB, Oyhenart EE. Prevalence of overweight and obesity among Guaraní-Mbyá from Misiones, Argentina. Am J Hum Biol 2006; 18:590-9.

23. Zeng W, Eisenberg DTA, Jovel KR, Undurraga EA, Nyberg C, Tanner S, et al. Adult obesity: panel study from native Amazonians. Econ Hum Biol 2013; 11:227-35.

24. Horta BL, Santos RV, Welch JR, Cardoso AM, Santos JV, Assis AM, et al. Nutritional status of indigenous children: findings from the First National Survey of Indigenous People's Health and Nutrition in Brazil. Int J Equity Health 2013; 12:23.

25. Leite MS, Cardoso AM, Coimbra Jr. CEA, Welch JR, Gugelmin SA, Lira PC, et al. Prevalence of anemia and associated factors among indigenous children in Brazil: results from the First National Survey of Indigenous People's Health and Nutrition. Nutr J 2013; 12:69.

26. Pinho CP, Diniz AS, Arruda IK, Lira PI, Sequeira LA, Gonçalves FC, et al. Excesso de peso em adultos do Estado de Pernambuco, Brasil: magnitude e fatores associados. Cad Saúde Pública 2011; 27:2340-50.

27. Lino MZR, Muniz PT, Siqueira KS. Prevalência e fatores associados ao excesso de peso em adultos: inquérito populacional em Rio Branco, Acre, Brasil, 2007-2008. Cad Saúde Pública 2011; 27: 797-810. 
28. Silva DA, Felisbino-Mendes MS, Pimenta AM, Gazzinelli A, Kac G, Velásques-Meléndez G. Distúrbios metabólicos e adiposidade em uma população rural. Arq Bras Endocrinol Metab 2008; 52:489-98.

29. Silva H, Padez C. Body size and obesity patterns in Caboclo populations from Pará, Amazonia, Brazil. Ann Hum Biol 2010; 37:217-29.

30. Gigante DP, Moura EC, Sardinha LM. Prevalence of overweight and obesity and associated factors, Brazil, 2006. Rev Saúde Pública 2009; 43 Suppl 2: 83-9.

31. Veloso HJF, Silva AUM. Prevalência e fatores associados à obesidade abdominal e ao excesso de peso em adultos maranhenses. Rev Bras Epidemiol 2010; 13:400-12.
32. World Health Organization. Obesity: preventing and managing the global epidemic. Geneva: World Health Organization; 2000. (Technical Report Series, 894).

33. Gugelmin SA, Santos RV. Uso do índice de massa corporal na avaliação do estado nutricional de adultos indígenas Xavánte, Terra Indígena Sangradouro-Volta Grande, Mato Grosso, Brasil. Cad Saú de Pública 2006; 22:1865-72.

Recebido em 31/Mai/2014

Versão final reapresentada em 09/Jan/2015

Aprovado em 21/Jan/2015 